\title{
PRIMITIVE DIVISORS ON TWISTS OF FERMAT'S CUBIC
}

\section{GRAHAM EVEREST, PATRICK INGRAM AND SHAUN STEVENS}

\section{Abstract}

We show that for an elliptic divisibility sequence on a twist of the Fermat cubic, $u^{3}+v^{3}=m$, with $m$ cube-free, all the terms beyond the first have a primitive divisor.

\section{Statement of Main Theorem}

Let $C$ denote a twist of the Fermat cubic,

$$
C: \quad U^{3}+V^{3}=m W^{3}
$$

with $m$ a non-zero rational number. If $K$ denotes any field of characteristic zero, the set $C(K)$ of projective $K$-rational points satisfying (1) forms an elliptic curve. With respect to the usual chord and tangent addition the set $C(K)$ forms a group. The identity of the group is $(-1,1,0)$ and the inverse of the point $(U, V, W)$ is $(V, U, W)$. Let $R \in C(\mathbb{Q})$ denote a non-torsion rational point. Write

$$
n R=\left(U_{n}, V_{n}, W_{n}\right), \quad U_{n}, V_{n}, W_{n} \in \mathbb{Z}
$$

in lowest form with $\operatorname{gcd}\left(U_{n}, V_{n}, W_{n}\right)=1$.

Definition 1.1. Let $\left(A_{n}\right)$ denote a sequence with integer terms. We say an integer $d>1$ is a primitive divisor of $A_{n}$ if

(a) $d \mid A_{n}$ and

(b) $\operatorname{gcd}\left(d, A_{m}\right)=1$ for all non-zero terms $A_{m}$ with $m<n$.

This paper is devoted to proving the following theorem.

TheOrem 1.2. Let $C$ denote the elliptic curve in (1) with $m \in \mathbb{Z}$ assumed to be cube-free. Let $W=\left(W_{n}\right)$ denote the sequence obtained as above from $R \in C(\mathbb{Q})$, a non-torsion rational point. For all $n>1$, the term $W_{n}$ has a primitive divisor.

The sequence $W=\left(W_{n}\right)$ is a divisibility sequence, which means that, for all $m, n \in \mathbb{N}$,

$$
m \mid n \text { implies } W_{m} \mid W_{n} \text {. }
$$

In line with recent developments $[\mathbf{1 1}, \mathbf{1 2}, \mathbf{1 3}, \mathbf{1 4}, \mathbf{1 5}, \mathbf{1 6}, \mathbf{2 5}, \mathbf{2 6}, \mathbf{2 7}]$ we define the sequence $W=\left(W_{n}\right)$ to be an Elliptic Divisibility Sequence. Admittedly this stretches the definition originally used by Morgan Ward [29] but we believe it is a reasonable name for a divisibility sequence that arises from an elliptic curve.

The research of the first and third authors was supported by EPSRC grant EP/E012590/1. The research of the second author is supported by a grant from the NSERC of Canada.

Received 19 February 2008, revised 2 September 2008; published 4 March 2009.

2000 Mathematics Subject Classification 11G05, 11A41

(C) 2009, Graham Everest, Patrick Ingram and Shaun Stevens 
In the sequel, it will often be convenient to work with the affine curve

$$
u^{3}+v^{3}=m
$$

and we will also refer to this curve as $C$. Properties of points such as being integral will generally refer to the affine curve. Thus, an integral point on $C$ is a pair of integers $(u, v)$ satisfying (3). Theorem 1.2 is the best possible in the sense that if $R$ is an integral point, then $W_{1}$ has no non-trivial divisors and so (by our definition) no primitive divisors. Thus, the identity

$$
(1+t)^{3}+(1-t)^{3}=\left(6 t^{2}+2\right)
$$

for any integer $t>1$, gives rise to an elliptic divisibility sequence for which Theorem 1.2 is best possible, whenever $6 t^{2}+2$ is cube-free. There are infinitely many $t$ such that $6 t^{2}+2$ is cube-free by a result of Erdös [9].

If one removes the condition that $m$ be cube-free, then it is easy to construct counter-examples to Theorem 1.2 by clearing denominators. However, any elliptic curve in the form (1) can be transformed into one with $m$ a cube-free integer by a simple scaling. Thus, Theorem 1.2 does give complete information, taking account of the transformation.

Note that Theorem 1.2 has an immediate application to the study of integral points on elliptic curves in the form (1). In [23], Silverman showed that, when $m$ is cube-free, there exists an absolute constant $\kappa$ such that $(1)$ has at most $\kappa^{1+\operatorname{rank}(C / \mathbb{Q})}$ integral points. In particular, the number of integral solutions to (3) is bounded uniformly if we restrict attention to curves $C$ with rank not exceeding a given bound. Note that $C(\mathbb{Q})$ is torsion-free when its $\operatorname{rank}$ is positive. Thus if $\operatorname{rank}(C / \mathbb{Q})=1$, then $C(\mathbb{Q})$ consists solely of the multiples of a single point $R$, say. By Theorem 1.2 , the denominator of $n R$, for each $n \neq \pm 1,0$, has a primitive divisor. In particular, none of these denominators is 1 .

Corollary 1.3. If $m \in \mathbb{Z}$ is cube-free, and $\operatorname{rank}(C / \mathbb{Q})=1$, then $(3)$ has at most two integral solutions - either of which generates $C(\mathbb{Q})$.

Corollary 1.3 is not overly surprising. The method of proof in [23] gives very strong bounds on the number of integral points on $C$ as the diophantine approximation involved in this case is trivial. Corollary 1.3 is noted because it is sharp and completely qualitative. For quantitative results of similar strength for other elliptic curves, see $[\mathbf{1 5}]$.

In the next section, we will set Theorem 1.2 in its proper context, as well as outline the structure of the proof. The proof occupies the rest of the paper.

\section{Primitive prime divisors}

Let $M=\left(M_{n}\right)$ denote the Mersenne sequence, whose $n$th term is $M_{n}=2^{n}-1$. No proof is known that $M$ contains infinitely many prime terms. The concept of a primitive divisor was introduced as a way of showing that new primes are produced by the terms of $M$, but in a less restrictive sense. In 1886 Bang [2] showed that if $a$ is any fixed integer with $a>1$ then the sequence with $n$th term $a^{n}-1$ has a primitive divisor for any index $n>6$. This is a sharp result because the term $M_{6}=63=M_{2}^{2} \cdot M_{3}$ does not have a primitive divisor. Bang's theorem is remarkable because the number 6 is uniform across all $a$ and it is small. Indeed, 
it is not hard to show that $a=2$ is the only example realizing this bound. Bang's Theorem was incredibly influential, as we now indicate briefly.

In 1892 Zsigmondy [30] obtained the generalization that for any relatively prime $a$ and $b$ with $a>b>0$, the terms of the sequence $A_{n}=a^{n}-b^{n}$ all have primitive divisors if $n>6$. This lovely result was re-discovered several times in the early 20 th century and it has turned out to be quite applicable. See [20] and the references therein where applications to Group Theory are discussed. For example, for fixed $q$ a prime power, let $\mathbb{F}_{q}$ denote the finite field with $q$ elements. Zsigmondy's Theorem applied to the explicit formula for the order of the group $G L_{n}\left(\mathbb{F}_{q}\right)$ shows this order has a primitive divisor for all large $n$. Thus Sylow's Theorem can be invoked to deduce information about the structure of the group.

Definition 2.1. Let $A=\left(A_{n}\right)_{n \geqslant 1}$ be an integer sequence. Define

$$
Z(A)=\max \left\{n \mid A_{n} \text { does not have a primitive divisor }\right\}
$$

if this set is finite, and $Z(A)=\infty$ if not. The number $Z(A)$ will be called the Zsigmondy bound for $A$.

Thus Bang's Theorem may be stated: $Z(M)=6$, while Zsigmondy's Theorem may be stated: $Z(A) \leqslant 6$, where $A_{n}=a^{n}-b^{n}$ as above. Following Zsigmondy's Theorem, the next major theoretical advance was made by Carmichael. Let $u$ and $v$ denote conjugate quadratic integers. Consider the integer Lucas sequence $U$ defined by

$$
U_{n}=\left(u^{n}-v^{n}\right) /(u-v) .
$$

For example, the Fibonacci sequence $F=\left(F_{n}\right)$ is a Lucas sequence. Carmichael [5] showed that if $u$ and $v$ are real then $Z(U) \leqslant 12$. This too is a sharp result because $F_{12}$ does not have a primitive divisor. The general case was settled by Bilu, Hanrot and Voutier [3]. They proved that $Z(U) \leqslant 30$ using a powerful cocktail of methods including start of the art bounds from Diophantine analysis as well as massive computations to deal with special cases. Again this is a sharp result as the sequence generated by the roots of the polynomial $x^{2}-x+2$ illustrates. The paper [3] gives details about the long journey from Carmichael's result to the general case.

\subsection{Elliptic curves}

Now let $E$ denote an elliptic curve in Weierstrass form,

$$
y^{2}+a_{1} x y+a_{3} y=x^{3}+a_{2} x^{2}+a_{4} x+a_{6}
$$

with $a_{1}, \ldots, a_{6} \in \mathbb{Z}$. The shape of the defining equation forces the denominator of $x(Q)$ to be an integer square, for any $Q \in E(\mathbb{Q})$. Let $Q \in E(\mathbb{Q})$ denote a non-torsion point. For every $n \in \mathbb{N}$ write

$$
x(n Q)=A_{n} / B_{n}^{2}
$$

in lowest terms. The sequence $B=\left(B_{n}\right)$ is an elliptic divisibility sequence associated to $Q$ and $E$. Silverman [28] obtained a primitive divisor theorem for elliptic divisibility sequences arising from curves in Weierstrass form. It seems likely that a uniform version of this theorem holds for curves in global minimal form. In other words, if $B=\left(B_{n}\right)$ arises from a rational point on a curve in global minimal form then $Z(B) \leqslant N_{0}$, where $N_{0}$ is independent of $E$ and $Q$. Indeed, the proof of 
Silverman's Theorem suggests that the Zsigmondy bound is higher for sequences generated by rational points with small global canonical height. Therefore it is significant that all such heights are conjectured (by Lang) to be uniformly bounded away from zero. This suggests that a proof that $Z(B)$ is uniformly bounded above seems to require a result as strong as Lang's conjecture. The following example appeared in $[\mathbf{1 4}]$.

EXAMPLE 2.2. Let $B$ denote the elliptic divisibility sequence generated by the point $Q=[7107,-602054]$ on the elliptic curve

$$
y^{2}+x y+y=x^{3}+x^{2}-125615 x+61201397
$$

Computations suggest that $Z(B)=39$ and no higher value of $Z(B)$ is known for an elliptic divisibility sequence coming from a Weierstrass curve in minimal form.

The curve in Example 2.2 was taken from a list of small height points maintained by Noam Elkies [8]. The curves in Elkies' table are not generally in minimal form but the curve in Example 2.2 has been rendered in minimal form in order to estimate $Z(B)$.

Computations with congruent number curves suggest the Zsigmondy bound is generally very small. A uniform Zsigmondy bound appears in [12] and [14] for an infinite class of sequences arising from congruent number curves. Specifically, let $T \geqslant 5$ denote a square-free integer and let $Q$ denote a non-torsion rational point on the curve

$$
y^{2}=x^{3}-T^{2} x .
$$

In [12] it was shown that, if $x(Q)<0$ or $x(Q)$ is a rational square, then $Z(B) \leqslant 21$. In [14] this result was improved by reducing the Zsigmondy bound to 2 (and allowing any of $x(Q)$ or $x(Q) \pm T$ to be a square), a bound witnessed by an infinite family of sequences. Provided the rank of the curve is positive, there will always be points satisfying the hypotheses stated: this is because, if $x(Q)>0$ then $x(Q+[0,0])<0$ and, for any rational point $Q$,

$$
x(2 Q) \text { and } x(2 Q) \pm T
$$

are all rational squares.

\subsection{Comparisons with the classical theory}

There are notable similarities between the results for elliptic divisibility sequences and the classical results described earlier. Both give a uniform bound across infinitely many sequences which is best possible, both rely upon lower bound on heights and both reduce the problem to solving a finite number of Thue-Mahler equations. Also, the uniformity result for Lucas sequences relies on good bounds from transcendence theory together with the fact that the answer to Lehmer's problem is known for quadratic integers. Therefore, a uniform result for elliptic divisibility sequences in general would appear to require not just Lang's conjecture on a uniform lower bound for heights of points, as we already mentioned, but also better elliptic transcendence results than are currently known.

In one respect, however, the arithmetic of these two classes of sequences differs markedly. Bang's Theorem may well have arisen as part of an attempt to prove the Mersenne Prime Conjecture (which remains open). On the other hand the analogue 
of that conjecture is false for elliptic divisibility sequences on curves of the form (1), and heuristics, as well as proofs in special cases, indicate that it fails for those on Weierstrass curves too, see $[\mathbf{1 0}]$ and $[\mathbf{1 1}]$.

In the section that follows we will reduce the given problem to one on the Weierstrass model of a curve birationally equivalent to the curve (1). The method proceeds in a pincer movement, somewhat similar to that in the two papers [12] and [14]. These papers used a good lower bound for the canonical height of a rational point which were obtained in [4]. Here, our workhorse is the paper [17], although the height bounds are not stated or used in the same way as in [17]. However, beside the similarities, there are many intriguing differences. Most notably, in this paper we make heavy use of a numerator sequence on a Mordell curve, see (4), for which we can prove a uniform Zsigmondy bound - see Theorem 2.3. Remarkably, this sequence is not a divisibility sequence, one of the few known cases where a primitive divisor theorem can be proved for a sequence which lacks the divisibility property. Also, remarkably, we have been unable to prove a uniform Zsigmondy bound for the corresponding denominator sequence on the Mordell curve.

\subsection{The structure of the proof}

The proof of Theorem 1.2 relies upon two different techniques. The first one bounds $Z(W)$ above by showing that the non-existence of a primitive divisor of $W_{n}$ implies a certain divisibility relation (see (24)) involving the terms $A_{n}$, which are defined in (5). This relation leads to an inequality which is violated for all sufficiently large $n$. This part results in an upper bound for $n$ of $n \leqslant 14$.

The second step shows directly that for each of the indices $n \geqslant 2$ not covered by the first part, $W_{n}$ does have a primitive divisor, by reducing the checking to a finite number of Thue-Mahler equations. In the reduction, the elliptic division polynomials, which are elliptic analogues of the cyclotomic polynomials, play a starring role.

The combination of techniques described here runs exactly parallel to those used in earlier primitive divisor theorems such as those of $[\mathbf{2}, \mathbf{3}, \mathbf{5}, \mathbf{3 0}]$. Note that it is essential to reduce the bound for $Z(W)$ in the first step as low as possible, in order to keep to a minimum the number of Thue-Mahler equations which need to be solved in the second step: without adequate care, computationally infeasible problems result.

The proof uses the well-known bi-rational equivalence of (3) with the Mordell curve

$$
E: Y^{2}=X^{3}-432 m^{2} \text {. }
$$

The map is given by

$$
u=\frac{36 m+Y}{6 X} \text { and } v=\frac{36 m-Y}{6 X} .
$$

If $R \in C(\mathbb{Q})$ corresponds to $Q \in E(\mathbb{Q})$ under the transformation, and we write

$$
n Q=\left(\frac{A_{n}}{B_{n}^{2}}, \frac{C_{n}}{B_{n}^{3}}\right),
$$

then

$$
\frac{U_{n}}{W_{n}}=\frac{36 m B_{n}^{3}+C_{n}}{6 A_{n} B_{n}} \text { and } \frac{V_{n}}{W_{n}}=\frac{36 m B_{n}^{3}-C_{n}}{6 A_{n} B_{n}} .
$$


The proof of Theorem 1.2 exploits both the denominator sequence $B=\left(B_{n}\right)$ in $(5)$, as in $[\mathbf{1 2}, \mathbf{1 4}]$, and the numerator sequence $A=\left(A_{n}\right)$. The latter may have independent interest, not least because it is a rare example where a primitive divisor result may be obtained, Theorem 2.3 below, for a sequence which is not a divisibility sequence. Instead, a weaker kind of divisibility relation holds, as detailed in Lemma 3.2.

TheOREm 2.3. Let $E$ denote the Mordell curve (4) and suppose $Q$ is a nontorsion point in $E(\mathbb{Q})$. Let $A=\left(A_{n}\right)$ denote the sequence as defined in $(5)$. Then $Z(A) \leqslant 12$.

In principle, techniques analogous to those used in [14] and Section 5 can be used here to reduce the bound stated in Theorem 2.3. In practice, however, the computations involved for some cases are beyond our current capabilities. We are uncertain about the supremum of the values $Z(A)$ as $m$ varies. Perhaps it occurs when $m=7$ : in this case $Z(A)=2$ because $A_{2}$ is a proper divisor of $A_{1}$, whereas all the terms $A_{3}, A_{4}, \ldots$ have a primitive divisor.

\section{Local arithmetic}

Since we are interested in the prime divisors of the numerators and denominators of $x(n Q)$, for $Q \in E(\mathbb{Q})$, it makes sense to address the local arithmetic of $E$.

Standing Assumption. Throughout Section 3, $p$ will be a prime other than 2 or 3 .

We will analyze $E\left(\mathbb{Q}_{p}\right)$, as in $[\mathbf{2 4}$, Chapter VII], through the exact sequence

$$
0 \rightarrow E_{1}\left(\mathbb{Q}_{p}\right) \rightarrow E_{0}\left(\mathbb{Q}_{p}\right) \rightarrow \tilde{E}_{\mathrm{ns}}\left(\mathbb{F}_{p}\right) \rightarrow 0
$$

where $E_{0}\left(\mathbb{Q}_{p}\right)$ is the subgroup of $E\left(\mathbb{Q}_{p}\right)$ consisting of points with nonsingular reduction modulo $p, E_{1}\left(\mathbb{Q}_{p}\right)$ is the kernel of reduction modulo $p$, and $\tilde{E}_{\mathrm{ns}}\left(\mathbb{F}_{p}\right)$ is the group of nonsingular points on the curve reduced modulo $p$. Throughout the paper, we adopt the familiar convention of referring to the term nonsingular reduction as good reduction, and the term singular reduction as bad reduction.

For an arbitrary integer sequence $A=\left(A_{n}\right)$, we follow Morgan Ward's terminology [29] and define the rank of apparition of the prime $p$ in the sequence $A$ to be the least index $n$ such that $p \mid A_{n}$ (the rank is $\infty$ if no such index exists). For the sequence $B$ defined above, note that $p \mid B_{n}$ is equivalent to $\operatorname{ord}_{p}(x(n Q))<0$, in other words, $n Q \in E_{1}\left(\mathbb{Q}_{p}\right)$. The rank of apparition of $p$ in the sequence $B$ is, then, the order of $Q$ in $E\left(\mathbb{Q}_{p}\right) / E_{1}\left(\mathbb{Q}_{p}\right)$, and $p \mid B_{k}$ precisely when $k$ is divisible by this order. In fact, the power to which $p$ divides $B_{k n}$ is entirely predictable once we know the power to which $p$ divides $B_{n}$. The following lemma is obtained in [24] by appeal to formal groups (and by an examination of division polynomials in [14]).

Lemma 3.1. Let $Q \in E_{1}\left(\mathbb{Q}_{p}\right)$. Then

$$
\operatorname{ord}_{p}(x(k Q))=\operatorname{ord}_{p}(x(Q))-2 \operatorname{ord}_{p}(k) .
$$

Remember that $p>3$ is a standing assumption in this section. Lemma 3.1 is not generally true when $p=2$ (although it is true when $p=3$ ). 
Note that the commentary above about the rank of apparition leads to an entirely algebraic interpretation of primitive divisors in the sequence $B$. To say that the term $B_{n}$ fails to have a primitive divisor is to say that there is no prime $p$ such that $Q$ has order exactly $n$ in $E\left(\mathbb{Q}_{p}\right) / E_{1}\left(\mathbb{Q}_{p}\right)$. This quotient, for primes $p$ of good reduction, is simply $\tilde{E}\left(\mathbb{F}_{p}\right)$. The rank of apparition of $p$ in the sequence $A$ may similarly be interpreted in terms of the local arithmetic of $E$, although the interpretation depends on whether $E$ has good or bad reduction at $p$.

If $p$ is a prime of bad reduction for $E$, then $p \mid m$ (recalling again that $p>3$ here). The curve $\tilde{E}$ has a singularity at the point $[0,0]$, and so $p \mid A_{n}$ precisely when $n Q$ is singular modulo $p$, in other words, $n Q$ has non-trivial image in the quotient $E\left(\mathbb{Q}_{p}\right) / E_{0}\left(\mathbb{Q}_{p}\right)$. Since the discriminant of $E$ is $\Delta(E)=-2^{12} 3^{9} m^{4}$, we see that $\operatorname{ord}_{p}(\Delta(E))$ is divisible by 4 for all $p \mid m$ other than 3 , and one can easily check (see [24, Table 15.1] or use the addition formula) that

$$
E\left(\mathbb{Q}_{p}\right) / E_{0}\left(\mathbb{Q}_{p}\right) \cong \mathbb{Z} / 3 \mathbb{Z}
$$

Thus if $p$ appears in the sequence $A$ at all, then it appears in precisely the terms $A_{k}$ for which $3 \nmid k$. The primes so occurring are, of course, exactly the primes of bad reduction dividing $A_{1}$.

If, on the other hand, $p \nmid m$, then $E\left(\mathbb{Q}_{p}\right)=E_{0}\left(\mathbb{Q}_{p}\right)$ and $\tilde{E}=\tilde{E}_{\mathrm{ns}}$. Let $H_{p} \subseteq \tilde{E}\left(\overline{\mathbb{F}}_{p}\right)$ be the subgroup generated by the two points in $\tilde{E}\left(\overline{\mathbb{F}}_{p}\right)$ with $x$-coordinate 0 . It is easy to verify that $H_{p} \cong \mathbb{Z} / 3 \mathbb{Z}$, and clearly $p \mid A_{n}$ if and only if

$$
n \tilde{Q} \in H_{p} \backslash\{\mathcal{O}\}
$$

where $\tilde{Q}$ is the image of $Q$ in $\tilde{E}\left(\mathbb{F}_{p}\right)$. Thus the rank of apparition of $p$ in $A$ is the order of $\tilde{Q}$ relative to $H_{p} \backslash\{\mathcal{O}\}$, or the least $n$ such that $n Q \in H_{p} \backslash\{\mathcal{O}\}$. Note that as $-432 m^{2}=-3(12 m)^{2}$, we have

$$
H_{p} \subseteq \tilde{E}\left(\mathbb{F}_{p}\right) \quad \text { if and only if } \quad p \equiv 1 \bmod 3,
$$

that is, precisely if $E$ has ordinary reduction at $p$. Super-singular primes cannot appear at all in the sequence $A$, in marked contrast with the situation for the sequence $B$, in which every prime eventually occurs. Of course, even $p$ being a prime of ordinary reduction for $E$ does not ensure that $p$ has finite rank of apparition in A.

The usefulness of Lemma 3.1, from our perspective, is that it allows one to obtain a strong bound on the size of a term $B_{n}$ failing to have a primitive divisor, as in $[\mathbf{1 2}, \mathbf{1 4}, \mathbf{1 6}]$. The main goal of this section is to prove a similar result for the sequence $A$.

Lemma 3.2. Suppose $\operatorname{ord}_{p}(x(Q))>0$. Then, for any $k$ coprime to 3 ,

$$
\operatorname{ord}_{p}(x(k Q))=\operatorname{ord}_{p}(x(Q))+\operatorname{ord}_{p}(k) .
$$

Proof. Much of what we need to prove our result has already been established. If $p$ is a prime of good reduction for $E$, then the condition $\operatorname{ord}_{p}(x(Q))>0$ ensures that $\tilde{Q} \in H_{p} \backslash\{\mathcal{O}\}$. In particular, $3 \tilde{Q}=\mathcal{O}$, and so $3 Q \in E_{1}\left(\mathbb{Q}_{p}\right)$. As $3 \nmid k$, we have $k \tilde{Q} \in H_{p} \backslash\{\mathcal{O}\}$ as well, and consequently $3 k Q \in E_{1}\left(\mathbb{Q}_{p}\right)$. Indeed, triplication on $E$ follows the law

$$
x(3 Q)=\frac{x^{9}(Q)+2^{9} 3^{4} x^{6}(Q) m^{2}+2^{12} 3^{7} x^{3}(Q) m^{4}-2^{18} 3^{9} m^{6}}{9 x^{2}(Q)\left(x^{3}(Q)-2^{6} 3^{3} m^{2}\right)^{2}},
$$


and so we deduce (for primes $p \nmid m$ ) that

$$
\operatorname{ord}_{p}(x(Q))>0 \Longrightarrow \operatorname{ord}_{p}(x(3 Q))=-2 \operatorname{ord}_{p}(x(Q))
$$

Applying Lemma 3.1, we obtain

$$
\begin{aligned}
2 \operatorname{ord}_{p}(x(k Q)) & =-\operatorname{ord}_{p}(x(3 k Q)) \\
& =-\operatorname{ord}_{p}(x(3 Q))+2 \operatorname{ord}_{p}(k) \\
& =2 \operatorname{ord}_{p}(x(Q))+2 \operatorname{ord}_{p}(k)
\end{aligned}
$$

The proof is somewhat more involved for the case where $p$ is a prime of bad reduction. In this case we know that $E\left(\mathbb{Q}_{p}\right) / E_{0}\left(\mathbb{Q}_{p}\right) \cong \mathbb{Z} / 3 \mathbb{Z}$, and $\operatorname{ord}_{p}(x(Q))>0$ ensures that $Q$ is non-trivial in this quotient. If we let

$$
E_{n}\left(\mathbb{Q}_{p}\right)=\left\{Q \in E\left(\mathbb{Q}_{p}\right): \operatorname{ord}_{p}(x(Q)) \leqslant-2 n\right\}
$$

then for each $n \geqslant 0$,

$$
E_{n}\left(\mathbb{Q}_{p}\right) / E_{n+1}\left(\mathbb{Q}_{p}\right) \cong \mathbb{Z} / p \mathbb{Z}
$$

This agrees with our definitions above of $E_{0}$ and $E_{1}$, and the fact that $E$ has additive reduction modulo $p$. It is worth mentioning that (9) is essentially equivalent to Lemma 3.1 (although (9) only holds in the general case for $n \geqslant 1$ ).

Returning to $(8)$ we see that $\operatorname{ord}_{p}(x(Q))>0$ implies

$$
2 \operatorname{ord}_{p}(x(Q))=-\operatorname{ord}_{p}(x(3 Q))+2 \operatorname{ord}_{p}(m)
$$

If $3 Q \in E_{n}\left(\mathbb{Q}_{p}\right) \backslash E_{n+1}\left(\mathbb{Q}_{p}\right)$ and $r=\operatorname{ord}_{p}(k)$, then (9) tells us that $3 k Q \in E_{n+r}\left(\mathbb{Q}_{p}\right) \backslash$ $E_{n+r+1}\left(\mathbb{Q}_{p}\right)$ so, by $(10)$,

$$
\begin{aligned}
2 \operatorname{ord}_{p}(x(k Q)) & =-\operatorname{ord}_{p}(x(3 k Q))+2 \operatorname{ord}_{p}(m) \\
& =-\operatorname{ord}_{p}(x(3 Q))+2 \operatorname{ord}_{p}(k)+2 \operatorname{ord}_{p}(m) \\
& =2 \operatorname{ord}_{p}(x(Q))+2 \operatorname{ord}_{p}(k) .
\end{aligned}
$$

4. The proofs that $Z(A) \leqslant 12$ and $Z(W) \leqslant 14$

After gathering some preliminary results, we proceed with the proof of Theorem 2.3. The proof that $Z(W) \leqslant 14$, which advances along similar lines, follows. The step from $Z(W) \leqslant 14$ to $Z(W) \leqslant 1$ is taken in Sections 5 and 6 .

\subsection{Preliminaries}

It will not generally be true that the defining equation (4) for $E$ is in global minimal form. The following comes from [17, Lemma 1]. 
Lemma 4.1. If $9 \mid m$ write $M=m / 9$. Then the global minimal form $E^{*}$ for $E$ is indicated:

$$
\begin{aligned}
& \text { (I) } \quad E^{*}: \quad y^{2}=x^{3}-\frac{27 m^{2}}{4} \quad \text { if } 2 \mid m \text { and } 9 \nmid m \\
& \text { (II) } \quad E^{*}: y^{2}+y=x^{3}-\frac{27 m^{2}+1}{4} \quad \text { if } 2 \nmid m \text { and } 9 \nmid m \\
& \text { (III) } E^{*}: \quad y^{2}=x^{3}-\frac{3 M^{2}}{4} \quad \text { if } 2 \mid m \text { and } 9 \mid m \\
& \text { (IV) } \quad E^{*}: y^{2}+y=x^{3}-\frac{3 M^{2}+1}{4} \quad \text { if } 2 \nmid m \text { and } 9 \mid m \text {. }
\end{aligned}
$$

The following explicit transformations render the curve $E$ in minimal form:

$$
X=u^{2} x \text { and } Y=u^{3} y+t
$$

where $[u, t]=[2,0],[2,4],[6,0],[6,108]$ (respectively).

In the sequel, these four possibilities will be referred to as Cases (I)-(IV).

LEMmA 4.2. Write $Q$ for a non-torsion point on $E(\mathbb{Q})$, corresponding to $Q^{*} \in E^{*}(\mathbb{Q})$, where $E^{*}$ denotes the minimal model. Write, for all $n \geqslant 1, x\left(n Q^{*}\right)=a_{n} / b_{n}^{2}$ and $h=\hat{h}(Q)$. Also, write $M=m / 9$ if $9 \mid m$ and $M=m$ otherwise. Then

$$
-\frac{2}{3} \log M-\frac{3}{2} \log 3 \leqslant h n^{2}-\frac{1}{8} \log \left|a_{n}^{4}+\frac{54 M^{2} a_{n} b_{n}^{6}}{m}\right| \leqslant \frac{1}{12} \log 3 .
$$

The proof follows immediately from [17, Proposition 2]. Note the misprint in [17] (which has + signs on the left hand side). We are going to use this in the following form:

$$
\begin{gathered}
h n^{2}-\frac{1}{12} \log 3-\frac{1}{8} \log \left|1+\frac{54 M^{2}}{m x_{n}^{3}}\right| \leqslant \frac{1}{2} \log a_{n} \text { and } \\
\frac{1}{2} \log a_{n} \leqslant h n^{2}+\frac{2}{3} \log M+\frac{3}{2} \log 3 .
\end{gathered}
$$

Lemma 4.3. Let $P$ denote any non-torsion point in $E(\mathbb{Q})$. Then

$$
\hat{h}(P) \geqslant \frac{1}{27} \log m-\frac{1}{27} \log 2-\frac{1}{36} \log 3>\frac{1}{27} \log m-.0562
$$

unless $m \equiv \pm 2 \bmod 9$ and $m$ has a prime factor congruent to $1 \bmod 6$, in which case

$$
\hat{h}(P) \geqslant \frac{1}{27} \log m-\frac{1}{27} \log 2-\frac{1}{12} \log 3 \geqslant \frac{1}{27} \log m-.1173
$$

REMARK 4.4. The difference between the bounds in (13) and (14) might seem so slight as to be hardly worth mentioning. However the sieving allowed by the second bound greatly reduces the amount of manual checking in the sequel. Having said that, it will become clear in the following proof that further sieving is possible. The lemma as stated represents a compromise between further savings on the checking of values of $m$ as against a more complicated version of Lemma 4.3. 
Proof. The proof of Lemma 4.3 uses the analysis in [17]. It begins by estimating a lower bound for $\hat{h}(k P)$, for $k=2$ or 3 , then uses $\hat{h}(k P)=k^{2} \hat{h}(P)$ to obtain the bound sought. The global height is bounded by estimating the local canonical height $\lambda_{p}$ at each place. Write

$$
\hat{h}(P)=\sum_{p \leqslant \infty} \lambda_{p}(P)
$$

We write $Q=k P$ and estimate $\lambda_{p}(Q)$ for each finite prime $p$. Suppose that $Q \in E_{0}\left(\mathbb{Q}_{p}\right)$, the non-singular part of the $p$-adic curve; then from $[\mathbf{1 7},(2.5)]$ we get

$$
\lambda_{p}(Q)=\frac{1}{2} \log \max \left\{1,|x(Q)|_{p}\right\}-\frac{1}{12} \log \left|\Delta^{*}\right|_{p},
$$

where $\Delta^{*}$ denotes the discriminant of the minimal equation, so

$$
\lambda_{p}(Q) \geqslant-\frac{1}{12} \log \left|\Delta^{*}\right|_{p} .
$$

For the archimedean valuation, assume first that $9 \nmid m$. In $[\mathbf{1 7},(2.3)]$ the following bound is proved:

$$
\lambda_{\infty}(Q) \geqslant \frac{1}{8} \log \left|x(Q)^{4}+54 m^{2} x(Q)\right|-\frac{1}{12} \log \Delta^{*} .
$$

The bound in (16) holds for any rational point.

If $Q \in E_{0}\left(\mathbb{Q}_{p}\right)$ for all primes $p$ then sum over all $p$, using (16) and (15), to obtain

$$
\hat{h}(Q) \geqslant \frac{1}{8} \log \left|x(Q)^{4}+54 m^{2} x(Q)\right|=\frac{1}{8} \log |x(Q)|+\frac{1}{8} \log \left|x(Q)^{3}+54 m^{2}\right|,
$$

using the product formula to write $\sum_{p \leqslant \infty} \log \left|\Delta^{*}\right|_{p}=0$. Since

$$
x(Q)^{3} \geqslant 27 m^{2} / 4
$$

it follows that

$$
\hat{h}(Q) \geqslant \frac{1}{24} \log \left(\frac{27}{4}\right)+\frac{1}{24} \log m^{2}+\frac{1}{8} \log m^{2}+\frac{1}{8} \log \left(\frac{27}{4}+54\right)>\frac{1}{3} \log m .
$$

If $m \not \equiv \pm 2 \bmod 9$ then, according to [17, Page 180], $3 P \in E_{0}\left(\mathbb{Q}_{p}\right)$ for all primes $p$ so we may put $Q=3 P$. The lower bound

$$
\hat{h}(P)>\frac{1}{27} \log m
$$

results, which is stronger than the lower bound (13). If $m \equiv \pm 2 \bmod 9$ but $p$ has no prime factors congruent to 1 mod 6 then, by [17, Page 180] again, we may take $Q=2 P$. This time we obtain the lower bound

$$
\hat{h}(P)>\frac{1}{12} \log m
$$

which, again, is stronger than the lower bound in (13).

When $9 \mid m$, we use $[\mathbf{1 7},(2.4)]$, which gives

$$
\lambda_{\infty}(Q) \geqslant \frac{1}{8} \log \left|x(Q)^{4}+6 M^{2} x(Q)\right|-\frac{1}{12} \log \Delta^{*},
$$


in place of $(16)$. Assuming again that $Q \in E_{0}\left(\mathbb{Q}_{p}\right)$ for all primes $p$ and summing over $p$, we obtain

$$
\hat{h}(Q) \geqslant \frac{1}{8} \log \left|x(Q)^{4}+6 M^{2} x(Q)\right|=\frac{1}{8} \log |x(Q)|+\frac{1}{8} \log \left|x(Q)^{3}+6 M^{2}\right| .
$$

When $9 \mid m, x(Q)^{3} \geqslant 3 M^{2} / 4$ so

$$
\hat{h}(Q) \geqslant \frac{1}{24} \log \left(\frac{3}{4}\right)+\frac{1}{24} \log M^{2}+\frac{1}{8} \log M^{2}+\frac{1}{8} \log \left(\frac{3}{4}+6\right),
$$

which simplifies, upon setting $M=m / 9$ to

$$
\hat{h}(Q) \geqslant \frac{1}{3} \log m-\frac{1}{4} \log 3-\frac{1}{3} \log 2 .
$$

Again by $\left[\mathbf{1 7}\right.$, Page 180], we have $3 P \in E_{0}\left(\mathbb{Q}_{p}\right)$ for all primes $p$ and putting $Q=3 P$ yields

$$
\hat{h}(P) \geqslant \frac{1}{27} \log m-\frac{1}{36} \log 3-\frac{1}{27} \log 2>\frac{1}{27} \log m-.0562
$$

Thus (13) holds when $9 \mid m$.

Finally consider the case when $m \equiv \pm 2 \bmod 9$ but $p$ has prime factors congruent to $1 \bmod 6$. In this case $Q=3 P \in E_{0}\left(\mathbb{Q}_{p}\right)$ for all primes $p \neq 3$. When $p=3$, use $[\mathbf{1 7},(2.6)]$ to obtain,

$$
\lambda_{3}(Q) \geqslant-\frac{3}{2} \log 3-\frac{1}{12} \log \left|\Delta^{*}\right|_{3} .
$$

Now sum over all $p$, using (15), (16) and (17), to obtain

$$
\hat{h}(Q) \geqslant \frac{1}{8} \log \left|x(Q)^{4}+54 m^{2} x(Q)\right|-\frac{3}{2} \log 3 .
$$

Since $x(Q)^{3} \geqslant 27 m^{2} / 4$,

$$
\hat{h}(Q) \geqslant \frac{1}{24} \log \left(\frac{27}{4}\right)+\frac{1}{24} \log m^{2}+\frac{1}{8} \log m^{2}+\frac{1}{8} \log \left|\frac{27}{4}+54\right|-\frac{3}{2} \log 3,
$$

which simplifies to

$$
\hat{h}(Q) \geqslant \frac{1}{3} \log m-\frac{1}{3} \log 2-\frac{3}{4} \log 3 .
$$

Writing $Q=3 P$ and dividing through by 9 gives the bound in (14).

\subsection{Proof of Theorem 2.3}

Assume that $n$ is an index such that $A_{n}$ has no primitive divisor; the following proof shows that $n \leqslant 12$. The proof comes in two steps. For the first step we will show that the assumption implies a divisibility statement of the following kind:

$$
A_{n} \mid 2^{\mu} 3^{\lambda} \rho(n) \prod_{q \mid n} A_{\frac{n}{q}}
$$

where the product is taken over primes, and where $\rho(n)$ denotes the product of the primes $q>3$ which divide $n$,

$$
\rho(n)=\prod_{3<q \mid n} q
$$


This step is crucial in the sequel. A little parsimony here greatly reduces the number of values $m$ which need to be checked manually.

To prove (19), let $p$ be any prime dividing $A_{n}$. Assuming $A_{n}$ has no primitive divisor, there is a term $A_{k}$ with $k<n$ such that $p \mid A_{k}$. Initially, suppose that $p \nmid 6$. If $\alpha$ is the rank of apparition of $p$ in the sequence $A$ then we know, by the discussion in Section 3, that $p \mid A_{k}$ if and only if $k=d \alpha$ for some $d$ prime to 3 . Since $\alpha<n$, there is some prime $q$, necessarily distinct from 3 , such that $q \alpha \mid n$, and hence $p \mid A_{n / q}$. Applying Lemma 3.2, we have

$$
\operatorname{ord}_{p}\left(A_{n}\right)=\operatorname{ord}_{p}\left(A_{\frac{n}{q}}\right)+\operatorname{ord}_{p}(q) \leqslant \operatorname{ord}_{p}\left(A_{\frac{n}{q}}\right)+1 .
$$

Now consider the possibilities when $p \mid 6$, subdividing according to the cases in Lemma 4.1, beginning with the simplest.

Case II: $2 \nmid m, 9 \nmid m$. For the case when $p=2$ notice that there are no rational points on the minimal model with $x \equiv 0 \bmod 2$. This is because the expression $\left(27 m^{2}+1\right) / 4$ is odd and the equation $y^{2}+y=1$ has no solutions in $\mathbb{F}_{2}$. Hence the maximum value of $\operatorname{ord}_{2}\left(A_{n}\right)$ is 2 , using the transformation $X=4 x$, and indeed this is the 2 -adic order of all the $A_{n}$. In other words we may may take $\mu=0$ in (19), unless $n$ is prime, in which case $\mu=2$.

Similarly, on the minimal model there are no points with $x \equiv 0 \bmod 3$. To see this, notice that $\left(27 m^{2}+1\right) / 4 \equiv-20 \bmod 81$, if $3 \mid m$, and the equation $y^{2}+y+20 \equiv 0 \bmod 81$ has no solutions. If $3 \nmid m$ then $\left(27 m^{2}+1\right) / 4 \equiv 7 \bmod 81$ but $y^{2}+y+7 \equiv 0 \bmod 81$ has no solutions. Again, using the transformation $X=4 x$, we see that $3 \mid A_{n}$ cannot hold and we may take $\lambda=0$ in (19).

The possibilities for the remaining cases can be summarized as follows:

Case I: $2 \mid m, 9 \nmid m$. If $4 \nmid m$ then $x \equiv 0 \bmod 2$ does not hold while if $4 \mid m$ then $x \equiv 0 \bmod 4$ does not hold. Using the transformation $X=4 x$, we see that $2 \leqslant \operatorname{ord}_{2}\left(A_{n}\right) \leqslant 3$ so we take $\mu=1$, unless $n$ is prime, in which case $\mu=3$. For the prime 3 , when $3 \nmid m$ we find $x \equiv 0 \bmod 3$ does not hold while if $3 \mid m$ we find $x \equiv 0 \bmod 9$ does not hold. Hence we may take $\lambda=0$ unless $n$ is prime, in which case $\lambda=1$.

Case III: $2|m, 9| m$. If $4 \nmid m$ then $x \equiv 0 \bmod 2$ does not hold while if $4 \mid m$ then $x \equiv 0 \bmod 4$ does not hold. Using the transformation $X=36 x$, we see that $2 \leqslant \operatorname{ord}_{2}\left(A_{n}\right) \leqslant 3$ so we take $\mu=1$ unless $n$ is prime, in which case $\mu=3$. For the prime 3 , we find $x \equiv 0 \bmod 3$ does not hold. Hence we may take $\lambda=0$ unless $n$ is prime, in which case $\lambda=2$ (from $X=36 x$ ).

Case IV: $2 \nmid m, 9 \mid m$. We find that $x \equiv 0 \bmod 2$ does not hold and neither does $x \equiv 0 \bmod 3$. Using the transformation $X=36 x$ gives $\mu=\lambda=0$ unless $n$ is prime, in which case $\mu=\lambda=2$.

Now that (19) is established, take logarithms:

$$
\log A_{n} \leqslant \log \rho(n)+\sum_{q \mid n} \log A_{\frac{n}{q}}+\mu \log 2+\lambda \log 3
$$

where the sum runs over primes $q$. In each case, going to the minimal model means we must substitute $A_{n}=u^{2} a_{n}$. This yields

$$
\log a_{n} \leqslant \log \rho(n)+\sum_{q \mid n} \log a_{\frac{n}{q}}+2 \log u(\omega(n)-1)+\mu \log 2+\lambda \log 3,
$$


where $\omega(n)$ denotes the number of distinct prime divisors of $n$.

In order to apply Lemma (4.2) we will need the following bound:

$$
\log \left|1+\frac{54 M^{3}}{m x_{n}^{3}}\right| \leqslant \log 9=2 \log 3 .
$$

To prove (21) note firstly that when $9 \nmid m$, we have $m=M$. Using either form of the minimal equation in Lemma 4.1, it follows that $x_{n}^{3} \geqslant 27 \mathrm{~m}^{2} / 4$ and the bound in (21) follows at once. When $9 \mid m, M=m / 9$ and Lemma 4.1 gives $x_{n}^{3} \geqslant 3 M^{2} / 4$. Now (21) comes out in exactly the same way.

Multiplying (11) by 2 gives

$$
2 h n^{2}-\frac{1}{6} \log 3-\frac{1}{4} \log \left|1+\frac{54 M^{3}}{m x_{n}^{3}}\right| \leqslant \log a_{n}
$$

and inserting (21) gives

$$
2 h n^{2}-\frac{2}{3} \log 3 \leqslant \log a_{n} .
$$

Inserting this into (20), we get

$$
2 h n^{2}-\frac{2}{3} \log 3 \leqslant \log \rho(n)+\sum_{q \mid n} \log a_{\frac{n}{q}}+2(\omega(n)-1) \log u+\mu \log 2+\lambda \log 3,
$$

On the other hand, multiplying (12) by 2 , replacing $n$ by $n / q$, and inserting into (22) gives

$$
\begin{aligned}
2 h n^{2}-\frac{2}{3} \log 3 & \leqslant \log \rho(n)+\sum_{q \mid n}\left(2 h\left(\frac{n}{q}\right)^{2}+\frac{4}{3} \log M+3 \log 3\right) \\
+ & 2(\omega(n)-1) \log u+\mu \log 2+\lambda \log 3 .
\end{aligned}
$$

Re-arranging gives

$$
\begin{gathered}
2 h n^{2}\left(1-\sum_{q \mid n} \frac{1}{q^{2}}\right) \leqslant \log \rho(n)+\frac{4}{3} \omega(n) \log M \\
+2(\omega(n)-1) \log u+\mu \log 2+\left(\lambda+3 \omega(n)+\frac{2}{3}\right) \log 3 .
\end{gathered}
$$

To ease the notation, write

$$
f(n)=1-\sum_{\substack{q \mid n \\ q \text { prime }}} \frac{1}{q^{2}}
$$

Since $M \leqslant m$ in each case, dividing by $\log m$ yields

$$
\frac{2 h n^{2}}{\log m} f(n) \leqslant \frac{4}{3} \omega(n)+\frac{\log \rho(n)+\omega(n) \log \left(27 u^{2}\right)+\log \left(2^{\mu} 3^{\lambda+\frac{2}{3}} / u^{2}\right)}{\log m} .
$$

Case II: $2 \nmid m, 9 \nmid m$.

In this case, $u=2$ and $\mu=\lambda=0$ unless $n$ is prime, in which case $\mu=2$ and $\lambda=0$. When $n$ is composite, assume $m \geqslant 40$. The bound (13) inserted into (23) 
now forces $n \leqslant 12$. Assuming $m \geqslant 290$, the bound (14) inserted into (23) forces $n \leqslant 12$. Thus we need to check all cases manually when $m \leqslant 40$ and all cases $m \leqslant 290$ when $m \equiv \pm 2 \bmod 9$ and $m$ has a prime divisor $\equiv 1 \bmod 6$. This will follow in the next section.

When $n$ is prime, $\omega(n)=1$ but we need to take $\mu=3$ and $\lambda=1$. When $m \geqslant 40,(13)$ and (23) force $n \leqslant 7$. When $m \geqslant 290,(14)$ and (23) force $n \leqslant 7$.

REMARK 4.5. To obtain these explicit upper bounds rigorously requires a little more than checking (23), because some of the functions that appear in (23) are not monotonic. In fact (23) implies a weaker inequality, where $\omega(n)$ is replaced by its upper bound of $\log n / \log 2, \rho(n)$ is replaced by its upper bound of $n$, and $f(n)$ is replaced by its lower bound of .547 . The resulting inequality yields a rigorous upper bound and the remaining cases can be checked manually. In none of the cases needed does this higher bound exceed 24 so the amount of extra checking is negligible.

Now we summarize the arguments in the other three cases:

Case I: $2 \mid m, 9 \nmid m$. Here $u=2$ and $\mu=1$ and $\lambda=0$ unless $n$ is prime, in which case $\mu=3, \lambda=1$. Applying Lemma 4.3 together with (23) as before gives the same bounds as the previous case with the same manual checking to be done.

Case III: $2|m, 9| m$. Here $u=6, \mu=1$ and $\lambda=0$ unless $n$ is prime, in which case, $\mu=3$ and $\lambda=2$. Inserting Lemma 4.3 into (23), the same bounds arise.

Case IV: $2 \nmid m, 9 \mid m$. Here $u=6$ and $\mu=\lambda=0$ unless $n$ is prime, in which case $\mu=2, \lambda=2$. Inserting into (23) and using Lemma 4.3 gives exactly the same bounds as before.

The proof of Theorem 2.3 is complete subject to checking various values of $m$; see Appendix A.1 for the details.

\subsection{Proof that $Z(W) \leqslant 14$}

Note that if a prime $p$ is a primitive divisor of a term $A_{n}$, where $n \geqslant 2$, then it cannot divide $B_{r}$ with $r<n$. We have seen that a prime of bad reduction which divides $A_{n}$, with $n>1$, also divides $A_{1}$, and so such a prime cannot be primitive divisor of $A_{n}$. If $p \geqslant 5$ is a primitive divisor of $A_{n}$ then $p$ is a prime of good reduction for $E$ and so, by the results in Section 3, the rank of apparition of $p$ in the sequence $B$ is $3 n$. When $p \mid 6$, the same conclusion follows by manual checking, using the same case-by-case analysis as in Section 4.2.

The proof that $Z(W) \leqslant 14$ runs along almost the same lines as the proof that $Z(A) \leqslant 12$. Suppose $p \geqslant 5$ is a primitive divisor of $A_{n}$, and suppose that $p^{a} \| A_{n}$. Then either $p$ divides $W_{n}$, in which case it is a primitive divisor, or $p^{a} \mid 36 m B_{n}^{3}+C_{n}$, according to (6). By the identity

$$
\left(36 m B_{n}^{3}+C_{n}\right)^{3}+\left(36 m B_{n}^{3}-C_{n}\right)^{3}=m\left(6 A_{n} B_{n}\right)^{3},
$$

which is simply (1), we have $p^{a} \mid 36 m B_{n}^{3}-C_{n}$, whence $p^{a} \mid 36 m B_{n}^{3}$. As $A_{n}$ is prime to $B_{n}$, and as $p \geqslant 5$, we have $p^{a} \mid m$. Thus $W_{n}$ fails to have a primitive divisor just in case the 'primitive part' of $A_{n}$ divides $m$, or

$$
A_{n} \mid m 2^{\mu} 3^{\lambda} \rho(n) \prod_{q \mid n} A_{\frac{n}{q}} .
$$


The arguments of the previous section now apply mutatis mutandis to show that

$$
\frac{2 h n^{2}}{\log m} f(n) \leqslant 1+\frac{4}{3} \omega(n)+\frac{\log \rho(n)+\omega(n) \log \left(27 u^{2}\right)+\log \left(2^{\mu} 3^{\lambda+\frac{2}{3}} / u^{2}\right)}{\log m} .
$$

When $n$ is composite, assume $m \geqslant 40$. The bound (13) inserted into (25) now forces $n \leqslant 14$ in all cases. Assuming $m \geqslant 290$, the bound (14) inserted into (25) forces $n \leqslant 14$. This completes the proof that $Z(W) \leqslant 14$, subject to checking various values of $m$. The values of $m$ required to complete the argument are checked in Appendix A.1.

Thus the proof of Theorem 1.2 follows once we establish that $W_{n}$ has a primitive divisor for all $2 \leqslant n \leqslant 14$. To that end we now turn.

\section{Primitive divisors in specific terms}

In the present section we concern ourselves with the question of, for fixed $n$, which sequences $W$ fail to have a primitive divisor in the $n$th term. For many small values of $n$ we can show that there are no such sequences, and this will bridge the gap between the work in Section 4 and the goal of Theorem 1.2. To ease notation, we will frequently write $D$ in place of $-432 \mathrm{~m}^{2}$.

Proposition 5.1. Let $W$ be a sequence as defined above. Then $W_{n}$ has a primitive divisor for each $2 \leqslant n \leqslant 14$.

The proof will come in several pieces. For $n \geqslant 5$ not divisible by 3 , the methods developed in [14] suffice to treat this problem. Although we make use of the special form of $D=-432 \mathrm{~m}^{2}$, much of the argument will work for general Mordell curves. Indeed, we reduce the proposition to the checking of finitely many elliptic divisibility sequences arising from Mordell curves, none of which turn out to be curves of the special form under consideration. When $n \geqslant 6$ is divisible by 3 some problems arise, but only a slight modification of the method of [14] is needed. The cases $n=4$, $n=3$, and $n=2$, treated in Section 6, are dispatched largely through ad hoc means, although the spirit of the proof remains the same.

\subsection{Division polynomials}

We exploit, as above, the short Weierstrass form of the equation, and will in fact show that $W_{n}$ has a primitive divisor coprime to $6 m$ for the $n$ listed in the lemma. We will consider the curves $C$ and $E$, as defined in (1) and (4) respectively, birationally equivalent by the map defined in (6). There are (see, for example, [24]) rational maps $\phi_{n}, \psi_{n}, \omega_{n} \in \mathbb{Q}(E)$, the function field of $E$, such that, for each $n \in \mathbb{Z}$ and $Q \in E(\mathbb{Q})$,

$$
n Q=\left(\frac{\phi_{n}(Q)}{\psi_{n}^{2}(Q)}, \frac{\omega_{n}(Q)}{\psi_{n}^{3}(Q)}\right)=\left(\frac{A_{n}}{B_{n}^{2}}, \frac{C_{n}}{B_{n}^{3}}\right) .
$$

In Section 4, we exploited the existence of primitive divisors in the sequence $A$. Here we use the properties of the division polynomial $\psi_{n}$, much as in [14], to establish the existence of primitive divisors in $W_{n}$. An approach employing the polynomials $\phi_{n}$ would be similarly successful, and would be in keeping with the flavour of Section 4, but such an approach also encounters serious computational difficulties. 
We will show that for all $n$ under consideration (save those treated later as special cases), there is a prime $p$ dividing $B_{n}$ such that $p \nmid 6 A_{k} B_{k}$ for any $k<n$. Such a prime will divide $W_{n}$, as $\operatorname{gcd}\left(B_{n}, C_{n}\right)=1$, but not $W_{k}$ for $k<n$. That is, $p$ will be a primitive divisor of $W_{n}$. It turns out that this amounts to showing that there is some prime $p \nmid \Delta(E)$ such that $\operatorname{ord}_{p}\left(F_{n}(Q)\right)>0$, for a certain $F_{n} \in \mathbb{Q}(E)$ constructed below. The hypothesis that there is no such prime will lead us to a non-trivial solution of a certain Thue-Mahler equation which depends on $n$ but, critically, not on $Q$ or $m$. By explicitly solving the Thue-Mahler equations in question, we will treat all bar three cases of Proposition 5.1.

As $\mathbb{Q}(E)=\mathbb{Q}(x, y)$, with $y^{2}=x^{3}+D$ (for fixed $D=-432 m^{2}$ ), we may write $\phi_{n}, \psi_{n}$, and $\omega_{n}$ as polynomials in $x$ and $y$, where $y$ occurs only to the first power. In fact (see [24]), we may write $\phi_{n}$ as a polynomial in $x$ alone, and either $\psi_{n}$ or $y^{-1} \psi_{n}$ similarly, as $n$ is odd or even respectively. Note that, in all cases, $\psi_{n}^{2}$ may be written as a polynomial in $x$, and we will view $\phi_{n}$ and $\psi_{n}^{2}$ as elements of $\mathbb{Q}[x]$. In fact, if $E[n]$ denotes the kernel of multiplication by $n$ in $E(\overline{\mathbb{Q}})$,

$$
\psi_{n}^{2}(Q)=n^{2} \prod_{\substack{T \in E[n] \\ T \neq \mathcal{O}}}(x(Q)-x(T)) .
$$

Note that each linear term on the right side occurs precisely twice, except those corresponding to $T \in E[2]$.

As our proof relies on the properties of $\psi_{n}$, it is incumbent upon us to make a few observations (these remarks are made as well in [14]). Note that if $3 \nmid n$, then $(x, y) \in E[n]$ implies both $x \neq 0$ and

$$
(x, y),(\zeta x, y),\left(\zeta^{2} x, y\right) \in E[n]
$$

where $\zeta$ is a primitive 3 rd root of unity. In particular, $\psi_{n}$ or $\psi_{n} / y$, depending on the parity of $n$, is a polynomial in $x^{3} ; \psi_{n}^{2} \in \mathbb{Q}\left[x^{3}\right] \subseteq \mathbb{Q}[x]$. When $3 \mid n$, on the other hand, $\psi_{n}^{2} \in x^{2} \mathbb{Q}\left[x^{3}\right]$.

Although $\psi_{n}$ depends on $m$, our stated aim is to construct, from each term $W_{n}$ without a primitive divisor, a solution to a Thue-Mahler equation that is independent of $m$. Note that the points on $y^{2}=x^{3}+D$ and those on $y^{2}=x^{3}+D^{\prime}$ are related by the scaling map

$$
(X, Y) \leftrightarrow\left(X\left(\frac{D^{\prime}}{D}\right)^{\frac{1}{3}}, Y\left(\frac{D^{\prime}}{D}\right)^{\frac{1}{2}}\right) .
$$

Thus, the dependence of (26) on $D$ is transparent. Along the same lines as [14], we observe that $\psi_{n}^{2}$ may be written, over $\mathbb{Z}$, as a binary form in $x^{3}$ and $4 D$. We will abuse notation somewhat, and denote this form by $\psi_{n}^{2}$ as well, so that

$$
\psi_{n}^{2}(Q)=\psi_{n}^{2}\left(x^{3}, 4 D\right) .
$$

In general, $\psi_{n}^{2} \in \mathbb{Q}(E)$ is not irreducible. Aside from being a square when $n$ is odd, the function has several obvious factors. Let $e_{\infty}(T)$ denote the order of the torsion point $T \in E(\overline{\mathbb{Q}})$, and let

$$
F_{n}^{2}(Q)=\epsilon^{2}(n) \prod_{\substack{T \in E(\overline{\mathbb{Q}}) \\ e_{\infty}(T)=n}}(x(Q)-x(T)),
$$


where

$$
\epsilon(n)= \begin{cases}p & \text { if } n=p^{a} \text { is a prime power } \\ 1 & \text { otherwise }\end{cases}
$$

It is clear from (26) that

$$
\psi_{n}^{2}=\prod_{d \mid n} F_{d}^{2}
$$

Note that, when $n \neq 2$, each term on the right of (27) occurs precisely twice, allowing us to define $F_{n} \in \mathbb{Q}[x]$ implicitly in this way.

The functions $F_{n}$ may be viewed as the elliptic analogues of the cyclotomic polynomials exploited in $[\mathbf{3}, \mathbf{5}, \mathbf{2 1}]$ to treat the analogous problem for Lucas sequences. By the same arguments as in the case of $\psi_{n}$, the product in (27) defines a binary form over $\mathbb{Z}$ in $x^{3}$ and $4 D$, at least when $n \neq 3$. We will, then, write $F_{n}\left(x^{3}, 4 D\right)$ for the product above. We will frequently pass between the rational function $F_{n}(Q)$ and the binary form $F_{n}\left(A_{1}^{3}, 4 D B_{1}^{6}\right)$. It is worth noting that, as $A_{1}$ and $B_{1}$ are coprime, the primes appearing to a positive power in $F_{n}(Q)$ occur to the same power in $F_{n}\left(A_{1}^{3}, 4 D B_{1}^{6}\right)$, except possibly those dividing $\epsilon(n)$.

\subsection{Division polynomials in finite fields}

Note that if $p$ is a prime of good reduction for $E$, then the equations from [24] define the division polynomials in the same way for $E\left(\mathbb{F}_{p}\right)$. In particular, if $e_{p}(Q)$ is the order of the image of the point $Q \in E(\mathbb{Q})$ in the group $E\left(\mathbb{F}_{p}\right)$, then $e_{p}(Q)$ is precisely the rank of apparition of the prime $p$ in the sequence $\left(\psi_{n}(Q)\right)_{n \geqslant 1}$, that is, the smallest $n$ such that $\operatorname{ord}_{p}\left(\psi_{n}(Q)\right)>0$. Equivalently, $e_{p}(Q)$ is the unique $n$ such that $\operatorname{ord}_{p}\left(F_{n}(Q)\right)>0$. Note, on the other hand, that $x(Q) \equiv 0 \bmod p$ implies that $Q$ is a point of order 3 in $E\left(\mathbb{F}_{p}\right)$. More generally, then, if $n$ is the rank of apparition of $p$ in the sequence $\left(\phi_{n}(Q)\right)_{n \geqslant 1}$, it follows that $e_{p}(Q)=3 n$. The converse may fail, of course, as there may be points of order 3 in $\tilde{E}\left(\mathbb{F}_{p}\right)$ other than those with $x=0$. The following observation will be useful.

Lemma 5.2. Let $Q \in E(\mathbb{Q})$, let $A_{n}$ and $B_{n}$ be defined as above, and let $p \nmid \Delta(E)$. Then the following hold:

(a) if $\operatorname{ord}_{p}\left(\psi_{n}(Q)\right)>0$, then $p \mid B_{n}$;

(b) if $\operatorname{ord}_{p}\left(\phi_{n}(Q)\right)>0$, then $p \mid A_{n}$;

(c) if $\operatorname{ord}_{p}\left(F_{n}(Q)\right)>0$ and $3 \nmid n$, then $p \mid B_{n}$ and $p \nmid 6 A_{k} B_{k}$ for $k<n$.

Proof. Note that results similar to (a) and (b) are derived in $[\mathbf{1}, \mathbf{1 4}, \mathbf{1 6}]$. In particular, the result in [1] is stronger: the hypothesis is merely that the reduction of $Q$ modulo $p$ is not singular. The lemma is demonstrated here for completeness.

As

$$
\frac{A_{n}}{B_{n}^{2}}=\frac{\phi_{n}(Q)}{\psi_{n}^{2}(Q)},
$$

the conclusions of (a) and (b) can only fail if $\operatorname{ord}_{p}\left(\phi_{n}(Q)\right)$ and $\operatorname{ord}_{p}\left(\psi_{n}(Q)\right)$ are simultaneously positive. If this is the case, then $\operatorname{ord}_{p}\left(\psi_{k}(Q)\right)>0$ for $k=n \pm 1$ as, by definition (see [24]),

$$
\phi_{n}=x \psi_{n}^{2}-\psi_{n-1} \psi_{n+1} .
$$


By an easy modification of Lemma 4.1 of [29] (for details on this modification see [16]), we have $\operatorname{ord}_{p}\left(\psi_{3}(Q)\right)>0$ and $\operatorname{ord}_{p}\left(\psi_{4}(Q)\right)>0$. Computing the resultants of the binary forms $\psi_{3}\left(x^{3}, 4 D\right)$ and $\psi_{4}^{2}\left(x^{3}, 4 D\right)$ in $\mathbb{Z}[D]$, we see that this can happen only if $p \mid 6 m$. That is, if $p \mid \Delta(E)$.

Now suppose $\operatorname{ord}_{p}\left(F_{n}(Q)\right)>0$. Part (a) ensures that $p \mid B_{n}$, and the image of $Q$ in $E\left(\mathbb{F}_{p}\right)$ has order exactly $n$. If $p \mid B_{k}$ for $k<n$, then $\operatorname{ord}_{p}\left(\psi_{k}(Q)\right)>0$, and hence $e_{p}(Q) \leqslant k<n$, a contradiction. Similarly, if $p \mid A_{k}$ then we have $\operatorname{ord}_{p}\left(\phi_{k}(Q)\right)>0$. Hence the prime $p$ has finite rank of apparition in the sequence $\left(\phi_{n}(Q)\right)_{k \geqslant 0}$, and so $n=e_{p}(Q)$ is divisible by 3 . We assumed it was not.

\subsection{Thue-Mahler equations}

The factors $F_{n}$ are the binary forms exploited in $[\mathbf{1 4}, \mathbf{1 6}]$ to show the existence of primitive divisors in specific terms of elliptic divisibility sequences arising from elliptic curves in short Weierstrass form. We will use a similar approach here to construct primitive divisors of the sequence $B$. The following, simple observation will be used repeatedly.

Claim 5.3. Let

$$
s=A_{1}^{3} / \operatorname{gcd}\left(A_{1}^{3}, 4 D\right), \quad t=4 D B_{1}^{6} / \operatorname{gcd}\left(A_{1}^{3}, 4 D\right) .
$$

Then for all primes $p \geqslant 5$ dividing $\operatorname{gcd}\left(A_{1}, D\right)$, we have

$$
\operatorname{ord}_{p}(s)>\operatorname{ord}_{p}(t)=0 .
$$

Furthermore, for all primes $p \geqslant 5$, the quantity $\operatorname{ord}_{p}(t)$ is even.

Proof of the Claim. Note that, as $m$ is cube-free, we have

$$
\operatorname{ord}_{p}(m) \in\{1,2\}
$$

for all $p \geqslant 5$ dividing $m$. Suppose that $p \mid A_{1}$ (and hence $p \nmid B_{1}$ ). If $\operatorname{ord}_{p}(m)=1$, then

$$
\operatorname{ord}_{p}\left(A_{1}^{3}\right) \geqslant 3>2=\operatorname{ord}_{p}\left(4 D B_{1}^{6}\right)=\operatorname{ord}_{p}\left(-432 m^{2}\right) .
$$

$\operatorname{Thus} \operatorname{ord}_{p}(s)>\operatorname{ord}_{p}(t)$.

If, on the other hand, $\operatorname{ord}_{p}(m)=2$, then $p^{3}$ divides the right-hand-side of

$$
C_{1}^{2}=A_{1}^{3}-432 m^{2} B_{1}^{6} \text {. }
$$

But then $p^{4}$ divides $A_{1}^{3}-432 m^{2} B_{1}^{6}$, as $p$ divides the left-hand-side of the above to an even power. Now $p^{2} \mid A_{1}$, hence

$$
\operatorname{ord}_{p}\left(A_{1}^{3}\right) \geqslant 6>4=\operatorname{ord}_{p}\left(-432 m^{2}\right),
$$

and again $\operatorname{ord}_{p}(s)>\operatorname{ord}_{p}(t)$. The fact that $\operatorname{ord}_{p}(t)=0$ simply follows from $s$ and $t$ being, by construction, relatively prime.

Now suppose that $p \geqslant 5$, and further that $\operatorname{ord}_{p}(t) \neq 0$. We have just shown that this ensures $p \nmid A_{1}$, and so

$$
\operatorname{ord}_{p}(t)=\operatorname{ord}_{p}\left(-432 m^{2}\right)=2 \operatorname{ord}_{p}(m) \equiv 0 \bmod 2 .
$$

We are now in a position to present the main tool in the proof of Proposition 5.1 for the values of $n \geqslant 5$ which are not divisible by 3 . The values $n=2,4$, and those values divisible by 3 , require a slightly more careful treatment. 
Lemma 5.4. Suppose that $W_{n}$ has no primitive divisor, for $n \geqslant 5$ not divisible by 3 . Then, for $s$ and $t$ defined as above, $F_{n}(s, t)$ is divisible only by primes dividing $6 \epsilon(n)$.

Proof. We have seen that if $p \nmid \Delta(E)$ and $\operatorname{ord}_{p}\left(F_{n}(Q)\right)>0$, then $p$ is a primitive divisor of $W_{n}$. Thus, if $W_{n}$ has no primitive divisor, we must have $\operatorname{ord}_{p}\left(F_{n}(Q)\right)>0$ only for $p \mid \Delta(E)$. Clearing denominators, we see that the only primes dividing the integer $F_{n}\left(A_{1}^{3}, 4 D B_{1}^{6}\right)$ are those of bad reduction for $E$ or, possibly, those dividing $\epsilon(n)$. If $p \nmid 6 \epsilon(n)$ is one such prime, then $p \mid D$. But then

$$
F_{n}\left(A_{1}^{3}, 4 D B_{1}^{6}\right) \equiv \epsilon(n) A_{1}^{3 \operatorname{deg} F_{n}} \bmod p .
$$

In particular, we can only have $p \mid F_{n}\left(A_{1}^{3}, 4 D B_{1}^{6}\right)$ if $p \mid A_{1}$. In the latter case we have $p \mid \operatorname{gcd}\left(A_{1}, D\right)$ whence, by Claim 5.3, we obtain $\operatorname{ord}_{p}(s)>\operatorname{ord}_{p}(t)=0$. Note that $F_{n}(0,1)= \pm 1$ as in $[\mathbf{1 4}]$ (see also the tables in Appendix A.2). Thus

$$
F_{n}(s, t) \equiv \pm t^{\operatorname{deg} F_{n}} \not \equiv 0 \bmod p .
$$

In particular, the prime divisors of $F_{n}(s, t)$ are at most those of $6 \epsilon(n)$ :

$$
F_{n}(s, t)= \pm 2^{\alpha} 3^{\beta} \epsilon(n)^{\gamma} \text {. }
$$

Note, for the purposes of impending computations, that we can say somewhat more. If $n=p^{a}$ is a prime power (and so $\epsilon(n)=p$ ), then $\operatorname{ord}_{p}\left(F_{n}(Q)\right)>0$ and $p \mid \Delta(E)$ together imply $p \mid m$. In this case $D \equiv 0 \bmod p^{2}$, ergo

$$
F_{n}\left(A_{1}^{3}, 4 D B_{1}^{6}\right) \equiv p A_{1}^{3 \operatorname{deg}\left(F_{n}\right)} \bmod p^{2} .
$$

Thus if $p \nmid A_{1}$, we have $\operatorname{ord}_{p}\left(F_{n}\left(A_{1}^{3}, 4 D B_{1}^{6}\right)\right)=1$. If, on the other hand, $p \mid A_{1}$, we have $\operatorname{ord}_{p}(s)>\operatorname{ord}_{p}(t)$, and so $p \nmid F_{n}(s, t)$. In the notation of the proof, then, we may take $\gamma \in\{0,1\}$. We will see, by examining the individual forms, that we can control the exponents of 2 and 3 by elementary means as well.

\subsection{Solving the Thue-Mahler equations}

We have reduced the proof of Proposition 5.1 to treating the special cases $n=2,4$ and $3 \mid n$, as well as solving a number of Thue-Mahler equations. Although the proof appears to require us to find all solutions to the Thue-Mahler equations

$$
F_{n}(s, t)=2^{\alpha} 3^{\beta} \epsilon(n)^{\gamma},
$$

we have already seen that we need only consider equations wherein $\gamma \in\{0,1\}$. Although we can, a priori, restrict the exponents $\alpha$ and $\beta$ by employing such techniques as lower bounds on linear forms in $p$-adic logarithms, it turns out that we may also do so by more elementary means.

For the various $n$ under consideration, consider $F_{n} \bmod 2$, and note that

$$
F_{n}(s, t) \equiv 1 \bmod 2
$$

whenever $\operatorname{gcd}(s, t)=1$ (note that these forms are available below for examination). Similarly, we may consider $F_{n} \bmod 3$ and reduce the possible values of $\beta$ to three: 0 , $\operatorname{deg}\left(F_{n}\right)$, or $\frac{3}{2} \operatorname{deg}\left(F_{n}\right)$. Thus, our Thue-Mahler equations may be reduced to 6 or 12 Thue equations (depending on whether or not $n$ is a prime power) of the form

$$
F_{n}(s, t)=(-1)^{\delta} 3^{\beta} \epsilon(n)^{\gamma},
$$


with $\delta, \gamma \in\{0,1\}$ and $\beta \in\left\{0, \operatorname{deg} F_{n}, \frac{3}{2} \operatorname{deg}\left(F_{n}\right)\right\}$.

We may also disregard several possible solutions in advance. For example, some of these equations possess a solution $(s, t)$ with $t=0$. Such a solution cannot arise from a pair $(s, t)$ as constructed in Claim 5.3, however, as this would necessitate either $m=0$ or $B_{1}=0$. Furthermore, we have from Claim 5.3 that $\operatorname{ord}_{p}(t)$ is even for all $p \geqslant 5$, and so we may ignore solutions $(s, t)$ that fail to have this property. As

$$
t \operatorname{gcd}\left(A_{1}^{3}, 4 D\right)=-432 m^{2} B_{1}^{6}<0
$$

and

$$
(4 s+t) \operatorname{gcd}\left(A_{1}^{3}, 4 D\right)=4\left(A_{1}^{3}+D B_{1}^{6}\right)=4 C_{1}^{2}>0,
$$

we may conclude as well that $t<0$ and $4 s+t>0$ (here, notice that $C_{1}=0$ only for points $Q$ of order 2, which are not the type under consideration). Any solution not satisfying these inequalities may be discarded as well. Solutions wherein $s=0$ correspond to points $Q$ of order 3 , and the solution $(s, t)=(1,-1)$ gives rise to the point $(12,36)$, a point of order 3 on $y^{2}=x^{3}-432$. We shall call solutions falling into the above categories expected.

A computation in PARI/GP [19] shows that there are no solutions to any of the Thue equations above, other than (possibly) these expected solutions. In the appendix, we list the binary forms $F_{n}$ for the various values of $n$, in order that the reader may confirm these findings. We should note that the Thue equation solver in PARI/GP assumes, by default, the truth of the Generalised Riemann Hypothesis. This default was overridden, and our results verified unconditionally. The most strenuous computation arises in the case $n=11$, in which a Thue-Mahler equation of degree 20 must be treated. (The binary form arising in the case $n=13$ is of greater degree but factors, and so the equations above may be treated by elementary means.) Even this computation, however, took well less than a minute (on a $1.83 \mathrm{GHz}$ MacBook with $512 \mathrm{MB}$ of RAM).

\subsection{The case $3 \mid n$, but $n \neq 3$}

Some care must be taken when $3 \mid n$ and $n \neq 3$, but the methods are not fundamentally different.

If $F_{n} \in \mathbb{Q}(E)$ is defined as above, where $n=3 k$, and if $p$ is a primitive divisor of $A_{k}$, then we have $\operatorname{ord}_{p}\left(F_{n}(Q)\right)>0$. It is not clear, then, that the primitive divisor of $B_{n}$ which we prove to exist by the method above fails to divide $A_{k}$. We must modify our argument to show that $B_{n}$ has a primitive divisor distinct from those coming from $A_{k}$. The binary form $F_{n}$ factors in this case, and we will show that it suffices to show that one of the factors has a prime divisor other than 2 or 3 .

Let $H \subseteq E(\overline{\mathbb{Q}})$ be the group

$$
\{(0, \sqrt{D}),(0,-\sqrt{D}), \mathcal{O}\} \cong \mathbb{Z} / 3 \mathbb{Z}
$$

Note that for $D=-432 m^{2}, H \subseteq E(\mathbb{Q}(\sqrt{-3}))$, although much of what is written here applies for all $D$. For $Q \in E(\overline{\mathbb{Q}})$, let $e(Q, H)$ denote the least $k$ such that 
$k Q \in H$, if one exists, and $e(Q, H)=\infty$ otherwise. Set, for $n$ divisible by 3 ,

$$
G_{n}^{2}(Q)=\prod_{\substack{T \in E(\overline{\mathbb{Q}}) \\ e(T, H)=n / 3}}(x(Q)-x(T)) .
$$

As $H$ is fixed by the action of Galois on $\overline{\mathbb{Q}}$, we have immediately that $G_{n} \in \mathbb{Q}(E)$. If $G_{n}$ vanishes at $Q$, then $A_{n}=0$, and so we see that $G_{n} \mid \phi_{n}$. If we define $H_{p} \subseteq E\left(\overline{\mathbb{F}}_{p}\right)$ to be the analogous subgroup (for $p \nmid \Delta(E)$ ), and $e_{p}(Q, H)$ to be the analogous value, then $e_{p}(Q, H)$ is the rank of apparition of $p$ in the sequence $A$, as discussed above. Arguments similar to those before show that $G_{n}$ may be written as a binary form in $x^{3}$ and $4 D$ with coefficients in $\mathbb{Z}$, and we will denote this form by $G_{n}(X, Y)$. For example,

$$
G_{9}(X, Y)=X^{3}-24 X^{2} Y+3 X Y^{2}+Y^{3} .
$$

Note that the roots of $G_{n}$ are points on $E(\overline{\mathbb{Q}})$ of order precisely $n$. Thus $G_{n}$ divides $F_{n}$. Let

$$
F_{n}(X, Y)=G_{n}(X, Y) \tilde{F}_{n}(X, Y) .
$$

LEMmA 5.5. Suppose that $W_{n}$ has no primitive divisor, for $3 \mid n$. Then $\tilde{F}_{n}(s, t)$ is $a\{2,3\}$-unit.

Proof. Suppose $p \nmid \Delta(E)$ and $\operatorname{ord}_{p}\left(\tilde{F}_{n}(Q)\right)>0$. It follows that $\operatorname{ord}_{p}\left(F_{n}(Q)\right)>0$, and so, just as in Lemma 5.2, we have $p \mid B_{n}$ and consequently $p \mid W_{n}$. We have, by the same argument as is Lemma 5.2 , that $p \nmid B_{k}$ for $k<n$. Suppose $p \mid A_{k}$, for some $k<n$, and suppose without loss of generality that $k$ is the least such value. Then we have $e_{p}(Q)=3 k$, and so $n=3 k$. We have as well that $e_{p}(Q, H)=n / 3$, so $\operatorname{ord}_{p}\left(G_{n}(Q)\right)>0$. But $\tilde{F}_{n}$ and $G_{n}$ can have no common roots modulo $p$. If $Q$ were such a root, then $x(n \tilde{Q}) \equiv 0 \bmod p$ while also $x(n \tilde{Q})^{3} \equiv-4 D \bmod p$, clearly impossible if $p \nmid D$. So we must not have $p \mid A_{k}$.

If, on the other hand, $p \mid \Delta(E)$ and $p \geqslant 5$, we have $p \mid m$. As above, we have $\operatorname{ord}_{p}(s)>\operatorname{ord}_{p}(t)=0$ and so, as $\tilde{F}_{n}(1, x)$ is monic, $p \nmid \tilde{F}_{n}(s, t)$. The result is proved.

Note that the lemma is true in the case $n=3$. The binary form $\tilde{F}_{3}$ has degree 1 , however, and so we cannot proceed in the same way as we will for $n \geqslant 6$. As in the case of Lemma 5.4, some care must be taken with the exponents. We note that $\tilde{F}_{n}(s, t) \equiv 1 \bmod 2$ for all relatively prime $s$ and $t$, while

$$
\operatorname{ord}_{3}\left(\tilde{F}_{n}(s, t)\right)= \begin{cases}0,3, \text { or } 5 & \text { if } n=6 \\ 0,9, \text { or } 13 & \text { if } n=9 \\ 0,12, \text { or } 18 & \text { if } n=12 .\end{cases}
$$

Computations in PARI/GP are as in the previous case, and reveal no unexpected solutions.

\section{6. $\quad$ Three special cases}

We have, thus far, shown that $W_{n}$ has a primitive divisor for all $n \geqslant 5$. We treat the cases $2 \leqslant n \leqslant 4$ here, completing the proof of Theorem 1.2. 


\subsection{The cases $n=3$ and $n=4$}

To treat the case $n=3$, we show that $A_{3}$ must have a prime divisor not dividing $6 m A_{1} A_{2}$. Such a divisor, being a prime of good reduction for $E$, must divide $W_{3}$, but cannot divide $W_{1}$ or $W_{2}$. Suppose, to the contrary, that $A_{3}$ has no prime divisor other than those dividing $6 m A_{1} A_{2}$. Comparing $\phi_{3}$ to $\psi_{2}$ and $\phi_{2}$, we see that then any prime $p$ with $\operatorname{ord}_{p}\left(\phi_{3}(Q)\right)>0$ must be a prime of bad reduction for $E$. If $p \geqslant 5$ is a divisor of $m$ we see, just as above, that

$$
\phi_{3}(s, t)=s^{3}-24 s^{2} t+3 s t^{2}+t^{3}
$$

is not divisible by $p$. So $\phi_{3}(s, t)$ is a $\{2,3\}$-unit. Exactly as in previous cases, we reduce this to

$$
s^{3}-24 s^{2} t+3 s t^{2}+t^{3}= \pm 3^{\beta},
$$

with $\beta \in\{0,3,4\}$, by considering the possible values of $\phi_{3}$ modulo 2 and 3 . Computation produces no unexpected solutions.

For the case $n=4$, we will show that $A_{4}$ has a prime divisor not dividing $6 m A_{1} A_{2} A_{3}$. As this divisor is a prime of good reduction for $E$, it divides $W_{4}$, witnessing that $W_{4}$ has a primitive divisor. We consider one particular factor of $\phi_{4}^{2}(X, Y)$. Let

$$
F_{4}^{*}(X, Y)=X^{4}-134 X^{3} Y-84 X^{2} Y^{2}-32 X Y^{3}-2 Y^{4} .
$$

One can verify that this binary form divides $\phi_{4}^{2}$, and (by computation of resultants) that $F_{4}^{*}\left(A_{1}^{3}, 4 D B_{1}^{6}\right)$ has no prime factors in common with $A_{1}, A_{2}, A_{3}, B_{1}, B_{2}, B_{3}$, save possibly some divisors of $\Delta(E)$. By a final application of Claim 5.3, we see that $F_{4}^{*}(s, t)$ is a $\{2,3\}$-unit. Solving the implied Thue-Mahler equations, one finds no unexpected solutions.

\subsection{The case $n=2$}

It remains to check that, for any $m$ and $Q$, the term $W_{2}$ has a primitive divisor. From (6) we have, in the notation of previous sections, that

$$
\frac{U_{2}}{W_{2}}=\frac{36 m \psi_{2}^{3}(Q)+\omega_{2}(Q)}{6 \phi_{2}(Q) \psi_{2}(Q)}
$$

where

$$
\phi_{2}=x\left(x^{3}-8 D\right), \quad \psi_{2}^{2}=4\left(x^{3}+D\right) .
$$

We suppose that every prime dividing $W_{2}$ also divides $W_{1}$, and hence $6 A_{1} B_{1}$. If $p \geqslant 5$ is any prime with

$$
\operatorname{ord}_{p}\left(\psi_{2}(Q)\right)=\operatorname{ord}_{p}\left(4 A_{1}^{3}+4 D B_{1}^{6}\right)>0,
$$

then $p$ is a primitive divisor of $W_{2}$, or $p \mid 6 m$. Assuming that the former is not the case, then, we see that $(4 s+t)$ is a $\{2,3\}$-unit, employing Claim 5.3 as in the cases above. That is, we know that $p \mid\left(4 A_{1}^{3}+4 D B_{1}^{6}\right)$ only if $p \mid \operatorname{gcd}\left(A_{1}, m\right)$, in which case $\operatorname{ord}_{p}(s)>\operatorname{ord}_{p}(t)$. In fact, we can say slightly more. Just as in Section 5.4, we may conclude from the inequality

$$
\left(4 A_{1}^{3}+4 D B_{1}^{6}\right)=4 C_{1}^{2}>0
$$

that $(4 s+t)$ is a positive $\{2,3\}$-unit. 
Now consider the primes dividing $A_{1}^{3}-8 D B_{1}^{6}$. Just as in the previous case, if $p \geqslant 5$ is a prime divisor of this expression, and $p$ is not a primitive divisor of $W_{2}$, then $p \mid m$. If this is the case, then another application of Claim 5.3 tells us that $(s-2 t)$ is a $\{2,3\}$-unit. Solving two linear equations yields

$$
\begin{aligned}
& 9 s=W_{1}+2 W_{2} \\
& 9 t=-4 W_{1}+W_{2},
\end{aligned}
$$

where $W_{1}$ and $W_{2} \in \mathbb{Z}$ are $\{2,3\}$-units, and $W_{2}>0$. It is (28) in which we are most interested. The second part of Claim 5.3 tells us that $\operatorname{ord}_{p}(t)$ is even for any $p \geqslant 5$. Specifically, then, $t=-d x^{2}$, for some positive $d \mid 6$ and some $x \in \mathbb{Z}$ (recall that $t<0$ as per Section 5.4). Equation (28) is now the representation of $-d x^{2}$ as a sum or difference of $\{2,3\}$-units. We also know that $\operatorname{gcd}\left(W_{1}, W_{2}\right) \mid 9$, as $\operatorname{gcd}(s, t)=1$. We may use this information to solve the above system of equations for all possible values of $s$ and $t$. First, a lemma.

Lemma 6.1. The only integral equations $a=b+c$ such that

(a) $c<0$;

(b) $b$ and $c$ are $\{2,3\}$-units;

(c) $\operatorname{gcd}(b, c)=1$; and

(d) $a, 2 a, 3 a$, or $6 a$ is a perfect square

are the following:

$$
\begin{aligned}
1^{2} & =2^{1}-1, & 7^{2} & =3^{4}-2^{5}, \\
1^{2} & =2^{2}-3^{1}, & 2.1^{2} & =3^{1}-1, \\
1^{2} & =3^{1}-2^{1}, & 2.2^{2} & =3^{2}-1, \\
1^{2} & =3^{2}-2^{3}, & 2.11^{2} & =3^{5}-1, \\
5^{2} & =3^{3}-2^{1}, & 3.1^{2} & =2^{2}-1 .
\end{aligned}
$$

Proof. Supposing that $a=b+c$ is one such equation, we may multiply both sides by sufficient powers of 2 and 3 to obtain an equation of the form

$$
q^{2}=r^{3}-2^{\mu} 3^{\nu}
$$

where $r$ is a $\{2,3\}$-unit. Thus, if we write $\mu=\mu_{0}+6 \mu_{1}$, where $0 \leqslant \mu_{0}<6$, and write $\nu=\nu_{0}+6 \nu_{1}$ similarly, we see that

$$
\left(\frac{r}{2^{2 \mu_{1}} 3^{2 \nu_{1}}}, \frac{q}{2^{3 \mu_{1}} 3^{3 \nu_{1}}}\right)
$$

is a $\{2,3\}$-integral point on the elliptic curve $Y^{2}=X^{3}-2^{\mu_{0}} 3^{\nu_{0}}$, with the additional property that $X$ is a $\{2,3\}$-unit. Using MAGMA [18] to find all $\{2,3\}$-integral points on each curve of this form (for $0 \leqslant \mu_{0}, \nu_{0}<6$ ), and tracing these points back to the original equations, we have our result.

It is now a simple matter to solve the above system of equations for all possible $s$ and $t$. If $\operatorname{gcd}\left(W_{1}, W_{2}\right)=9$, then

$$
-t=\frac{4}{9} W_{1}-\frac{1}{9} W_{2}
$$

is an equation as in the lemma. Moreover, as $\frac{4}{9} W_{1}$ is divisible by 4 and $W_{2}>0$, most of the equations listed in the lemma may be disqualified immediately. We are 
left with the possibilities $\left(W_{1}, W_{2}\right)=(9,27)$ or $(9,9)$. These yield $(s, t)=(7,-1)$ or $(3,-3)$, the latter of which may be discarded as $\operatorname{gcd}(s, t)=1$ by construction.

If $\operatorname{gcd}\left(W_{1}, W_{2}\right)=3$, then

$$
-3 t=\frac{4}{3} W_{1}-\frac{1}{3} W_{2}
$$

defines an equation as in the lemma, yielding again the solution $(3,-3)$ for $(s, t)$. The case $\operatorname{gcd}\left(W_{1}, W_{2}\right)=1$ yields no new solution.

The only solution, then, that might contradict our claim, is that corresponding to $(s, t)=(7,-1)$. One may trace this solution back to the point $(2,-1)$ on the curve $u^{3}+v^{3}=7$. While the terms $A_{1}^{3}+D B_{1}^{6}$ and $A_{1}^{3}-8 D B_{1}^{6}$ do both turn out to be $\{2,3\}$-units in this example, we may simply compute $W_{1}=1$ and $W_{2}=3$ to see that $W_{2}$ does, indeed, have a primitive divisor.

This completes the proof of Proposition 5.1, and hence the proof of Theorem 1.2.

\section{Appendix A. Computations}

In this section we discuss some of the particulars of the computations.

\section{Appendix A.1. Small values of $m$}

As required in Sections 4.2 and 4.3, we computed manually the Zsigmondy bounds $Z(A)$ and $Z(W)$ for all the cases needed with $m \leqslant 290$. From the analysis given in Section 4.2 , it is sufficient to consider all $m \leqslant 40$ (in fact we go out to 50 ) but only those $40<m<290$, for which $m \equiv \pm 2 \bmod 9$ and which possess a prime divisor congruent to $1 \bmod 6$. In all cases when the rank is positive the rational torsion group is trivial.

For rank-1 curves, the values of $Z(W)$ are given in Table 1 . The values of $Z(A)$ are much simpler: $Z(A)=0$ except in the case $m=7$, when $Z(A)=2$.

Table 1: Zsigmondy bounds in rank-1

\begin{tabular}{|c|c|c||c|c|c|}
\hline$m$ & $E$-generator & $Z(W)$ & $m$ & $E$-generator & $Z(W)$ \\
\hline 6 & {$[28,80]$} & 0 & 7 & {$[84,756]$} & 1 \\
9 & {$[36,108]$} & 1 & 12 & {$[52,280]$} & 0 \\
13 & {$[52,260]$} & 0 & 15 & {$[49,143]$} & 0 \\
17 & {$[84,684]$} & 0 & 20 & {$[84,648]$} & 0 \\
22 & {$[553 / 9,4085 / 27]$} & 0 & 26 & {$[156,1872]$} & 1 \\
28 & {$[84,504]$} & 1 & 31 & {$[217,3131]$} & 0 \\
33 & {$[97,665]$} & 0 & 34 & {$[2733,4455]$} & 0 \\
35 & {$[84,252]$} & 1 & 42 & {$[172,280]$} & 0 \\
43 & {$[129,1161]$} & 0 & 49 & {$[196,2548]$} & 0 \\
50 & {$[8148 / 27,138736 / 27]$} & 0 & 51 & {$[5473 / 36,333935 / 216]$} & 0 \\
58 & {$[9444 / 27,173600 / 27]$} & 0 & 61 & {$[732,19764]$} & 1 \\
79 & {$[316,5372]$} & 0 & 97 & {$[388,7372]$} & 0 \\
133 & {$[228,2052]$} & 1 & 151 & {$[4228 / 9,261532 / 27]$} & 0 \\
169 & {$[2028,91260]$} & 1 & 223 & {$[1561 / 4,49283 / 8]$} & 0 \\
241 & {$[6748,554300]$} & 1 & 259 & {$[777,20979]$} & 0 \\
277 & {$[5817 / 4,441261 / 8]$} & 0 & 286 & {$[588,12960]$} & 1 \\
\hline
\end{tabular}


In rank-2 the situation is slightly more complicated. Provided $\hat{h}(Q)>0.1$, equation (23) yields the Zsigmondy bound $Z(A) \leqslant 12$, and equation (25) yields the Zsigmondy bound $Z(W) \leqslant 14$. In all cases, there were no non-trivial rational points with $\hat{h}(Q) \leqslant 0.1$ so no further checking was necessary. In Table 2 we list generators for each of the rank-2 curves in the range. These were looked up in Cremona's tables [7], when the conductor was below $10^{4}$, or computed using MAGMA [18] for larger conductors.

Table 2: Rank-2 generators

\begin{tabular}{|c|c|}
\hline$m$ & $E$-generators \\
\hline 19 & {$[156,1908],[228,3420]$} \\
30 & {$[76,224],[124,1232]$} \\
37 & {$[84,36],[148,1628]$} \\
65 & {$[129,567],[156,1404]$} \\
124 & {$[372,6696],[2356,114328]$} \\
182 & {$[273,2457],[364,5824]$} \\
209 & {$[1596,63612],[532,11476]$} \\
218 & {$[1308,47088],[13881 / 25,1534221 / 125]$} \\
254 & {$[16257 / 4,2072385 / 8],[508,10160]$} \\
\hline
\end{tabular}

Table 3: The binary forms $F_{n}$

\begin{tabular}{|c|c|}
\hline$F_{5}$ & {$[5,95,-15,-25,-1]$} \\
\hline$F_{7}$ & {$[7,986,-2681,-12964,3626,-1519,-686,-49,1]$} \\
\hline$F_{8}$ & {$[2,616,-7336,-1544,-3430,-4124,-952,-104,-1]$} \\
\hline$F_{10}$ & $\begin{array}{r}{[1,1173,-55284,29380,-368055,-1404072,-862941,542232,} \\
\ldots-104805,-7070,-474,-177,1]\end{array}$ \\
\hline$F_{11}$ & $\begin{array}{r}{[11,23221,-1153603,-62045313,66133914,-1596123771,} \\
\ldots-8579472693,-4760052033,-22319781,8054721004, \\
\ldots .10595519759,4869514969,1106263389,189881835, \\
\ldots .59389374,17393277,2270301,102729,605,-242,-1]\end{array}$ \\
\hline$F_{13}$ & $\begin{array}{r}{[13,74737,-10304874,-1459820466,7383882519,-294761888811,} \\
\ldots-3649379851026,-327751614216,3634612800273, \\
\ldots 75587434125411,206422282971957,165623202699903, \\
\ldots 77423927253309,50317031121903,70684315657137, \\
\ldots 64207462488471,30461492791431,8167061938581, \\
\ldots 1237534488021,33446767107,-47530886481,-16133119236, \\
\ldots-2480541102,-183218139,-6445998,-217503, \\
\ldots-22815,-338,1]\end{array}$ \\
\hline$F_{14}$ & $\begin{array}{r}{[1,8826,-3182349,27544616,-1267563423,-29876807793,} \\
\ldots-73452197357,-534368475927,-321414204609, \\
\ldots-159623734993,-250499094747,-930524257131, \\
\ldots-1172171589176,-509647490898,-20486729571, \\
\ldots 61406271479,22270327506,3403598121,263510632, \\
\ldots .15278739,2663808,488510,19851,537,1]\end{array}$ \\
\hline
\end{tabular}


Table 4: The binary forms $\tilde{F}_{n}$

\begin{tabular}{|l|l|}
\hline$\tilde{F}_{6}$ & {$[1,57,3,1]$} \\
$\tilde{F}_{9}$ & {$[1,657,6111,-3318,19647,12033,3972,684,9,1]$} \\
$\tilde{F}_{12}$ & {$[1,3630,-28608,392908,212553,1121508,168108,62712$,} \\
& $\ldots 69507,32782,3684,12,1]$ \\
\hline
\end{tabular}

\section{Appendix A.2. Division polynomials}

Various specific binary forms are used for computations in Section 5, and these are reproduced in Tables 3 and 4 . To save space, only the coefficients have been recorded. In the tables the line

$$
\begin{array}{|l|l|}
\hline F & {\left[v_{d}, v_{d-1}, \cdots, v_{1}, v_{0}\right]} \\
\hline
\end{array}
$$

is to be interpreted as the statement

$$
F(X, Y)=\sum_{i=0}^{d} v_{i} X^{i} Y^{d-i}
$$

Note that, as a result of the action of complex multiplication on $E$, the polynomial $\psi_{p}$ factors when $p \equiv 1 \bmod 3$. In actual computations, this fact can be exploited to great advantage, allowing the corresponding Thue equations to be solved by elementary means.

\section{References}

1. M. Ayad, 'Points $S$-entiers des courbes elliptiques', Manuscripta Math. 76 (1992) 305-324. 70

2. A. S. BANG, 'Taltheoretiske Undersøgelser', Tidskrift f. Math. 5 (1886) 70-80, 130-137. 55, 58

3. Y. Bilu, G. Hanrot and P. M. Voutier, 'Existence of primitive divisors of Lucas and Lehmer numbers'. With an appendix by M. Mignotte, J. reine angew. Math. 539 (2001) 75-122. 56, 58, 70

4. A. Bremner, J. H. Silverman and N. Tzanakis, 'Integral points in arithmetic progression on $y^{2}=x\left(x^{2}-n^{2}\right)^{\prime}$, J. Number Theory 80 (2000) 187-208. 58

5. R. D. Carmichael, 'On the numerical factors of the arithmetic forms $\alpha^{n} \pm b^{n}$, Ann. of Math. (2) 15 (1914) 49-70. 56, 58, 70

6. D. V. Chudnovsky and G. V. Chudnovsky, 'Sequences of numbers generated by addition in formal groups and new primality and factorization tests', Adv. in Appl. Math. 7 (1986) 385-434.

7. J. E. Cremona, 'Elliptic Curve Data up-dated 05-01-2007', www.warwick.ac.uk/staff/J.E.Cremona/ftp/data/INDEX.htm 78

8. N. ElKies, 'Rational points with low canonical height', http://www.math.harvard.edu/ elkies/low_height.html 57 
9. P. ERDös, 'Arithmetical properties of polynomials', J. London Math. Soc. 28 (1953) 416-425. 55

10. G. Everest, V. Miller and N. Stephens, 'Primes generated by elliptic curves', Proc. Amer. Math. Soc. 132 (2004) 955-963. 58

11. G. Everest and H. King, 'Prime powers in elliptic divisibility sequences', Math. Comp. 74 (2005) 2061-2071. 54, 58

12. G. Everest, G. McLaren and T. Ward, 'Primitive divisors of elliptic divisibility sequences', J. Number Theory 118 (2006) 71-89. 54, 57, 58, 59, 60

13. G. R. Everest, S. Stevens, D. Tamsett and T. Ward, 'Primes generated by recurrence sequences', American Mathematical Monthly, May 2007 (American Mathematical Society, Providence, RI). 54

14. P. IngRAm, 'Elliptic divisibility sequences over certain curves', J. Number Theory 123 (2007) 473-486. 54, 57, 58, 59, 60, 68, 69, 70, 71, 72

15. P. InGRAm, 'Multiples of integral points on elliptic curves', arXiv:math.NT/0802.2651v2. 54, 55

16. P. InGRAm and J. H. Silverman, 'Uniform estimates for primitive divisors in elliptic divisibility sequences', to appear in a forthcoming memorial volume for Serge Lang (Springer). 54, 60, 70, 71

17. T. JedrzeJAK, 'Height estimates on cubic twists of the Fermat elliptic curve', Bull. Austral. Math. Soc. 72 (2005) 177-186. 58, 61, 62, 63, 64

18. MAGMA, version V2.13, University of Sydney, http://magma.maths.usyd.edu.au/magma/index.html. 76, 78

19. PARI/GP, version 2.3.0, Bordeaux, 2005, http://pari.math.u-bordeaux.fr/. 73

20. C. Praeger, 'Primitive prime divisor elements in finite classical groups', Groups St. Andrews 1997 in Bath II (Cambridge University Press, 1999) 605-623. 56

21. A. Schinzel, 'Primitive divisors of the expression $A^{n}-B^{n}$ in algebraic number fields', J. reine angew. Math. 268/269 (1974) 27-33. 70

22. T. ShOREY and R. Tijdeman, Exponential diophantine equations, Cambridge Tracts in Mathematics 87 (Cambridge University Press, 1986).

23. J. H. Silverman, 'Integer points and the rank of Thue elliptic curves', Invent. Math. 66 (1982) 395-404. 55

24. J. H. Silverman, 'The arithmetic of elliptic curves', Graduate Texts in Mathematics 106 (Springer, New York, 1986). 59, 60, 68, 69, 70

25. J. H. Silverman, 'Common divisors of elliptic divisibility sequences over function fields', Manuscripta Mathematica 114 (2004) 431-446. 54

26. J. H. Silverman and N. Stephens, 'The sign of an elliptic divisibility sequence', J. Ramanujan Math. Soc. 21 (2006) 1-17. 54

27. J. H. Silverman, ' $p$-adic properties of division polynomials and elliptic divisibility sequences', Math. Ann. 332 (2005) 443-471. 54

28. J. H. Silverman, 'Wieferich's criterion and the abc-conjecture', J. Number Theory 30 (1988) 226-237. 56 
29. M. WARD, 'Memoir on elliptic divisibility sequences', Amer. J. Math. 70 (1948) 31-74. 54, 59, 71

30. K. Zsigmondy, 'Zur Theorie der Potenzreste', Monatsh. Math. 3 (1892) 265-284. 56, 58

Graham Everest g.everest@uea.ac.uk

School of Mathematics, University of East Anglia, Norwich NR4 7TJ, UK

Patrick Ingram pingram@math.utoronto.ca

Department of Mathematics, University of Toronto, Canada M5S 2E4

Shaun Stevens shaun.stevens@uea.ac.uk

School of Mathematics, University of East Anglia, Norwich NR4 7TJ, UK 